\title{
Plasmacytoid variant of bladder cancer defines patients with poor prognosis if treated with cystectomy and adjuvant cisplatin-based chemotherapy
}

Bastian Keck ${ }^{1 *}$, Sven Wach ${ }^{1}$, Robert Stoehr ${ }^{2}$, Frank Kunath ${ }^{1}$, Simone Bertz ${ }^{2}$, Jan Lehmann ${ }^{3}$, Michael Stöckle ${ }^{4}$, Helge Taubert ${ }^{1}$, Bernd Wullich ${ }^{1}$ and Arndt Hartmann ${ }^{2}$

\begin{abstract}
Background: Since the definition of different histologic subtypes of urothelial carcinomas by the World Health Organization (WHO) 2004 classification, description of molecular features and clinical behavior of these variants has gained more attention.

Methods: We reviewed 205 tumor samples of patients with locally advanced bladder cancer mainly treated within the randomized AUO-AB05/95 trial with radical cystectomy and adjuvant cisplatin-based chemotherapy for histologic subtypes. 178 UC, 18 plasmacytoid (PUC) and 9 micropapillary (MPC) carcinomas of the bladder were identified. Kaplan Meier analysis and backward multivariate Cox's proportional hazards regression analysis were performed to compare overall survival between the three histologic subtypes.

Results: Patients suffering from PUC have the worst clinical outcome regarding overall survival compared to conventional UC and MPC of the bladder that in turn seem have to best clinical outcome (27.4 months, 62.6 months, and 64.2 months, respectively; $\mathrm{p}=0.013$ by Kaplan Meier analysis). Backward multivariate Cox' $\mathrm{s}$ proportional hazards regression analysis (adjusted to relevant clinicopathological parameters) showed a hazard ratio of 3.2 $(p=0.045)$ for PUC in contrast to patients suffering from MPC.
\end{abstract}

Conclusions: Histopathological diagnosis of rare variants of urothelial carcinoma can identify patients with poor prognosis.

Keywords: Chemotherapy, Cystectomy, Micropapillary, Plasmacytoid, Prognosis, Urinary bladder neoplasm

\section{Background}

Urothelial carcinoma (UC) is one of the most common malignancies with a total of 110,500 new cases diagnosed per year in Western Europe [1]. The plasmacytoid urothelial carcinoma of the bladder (PUC) and the micropapillary carcinoma of the bladder (MPC) represent two of several rare variants of urothelial carcinoma, which were included in the World Health Organisation (WHO) classification in 2004 [2]. Each of these subtypes accounts for approximately $3-5 \%$ of UC in patients with muscle

\footnotetext{
* Correspondence: bastian.keck@uk-erlangen.de

'Department of Urology, University Erlangen, Krankenhausstraße 12, Erlangen 91054, Germany

Full list of author information is available at the end of the article
}

infiltration. Morphologically, PUC presents with a discohesive, single cell growth pattern, with eccentrically located nuclei and an abundant eosinophilic cytoplasm (Figure 1) [3]. PUC is usually diagnosed at an advanced pathological stage and survival appears to be more unfavourable to what has been described for conventional UC [4]. MPC was first described by Amin et al. in 1994 [5] and is characterised by medium sized cells surrounded by an eosinophilic cytoplasm with an irregular distribution of chromatin, frequent mitotic figures and nuclear pleomorphisms [5-8]. Because of this original identification, multiple reports describing the aggressive biologic behaviour of MPC and its unfavourable clinical course, in mainly small cohorts, have been published. Clinical data

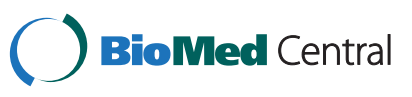




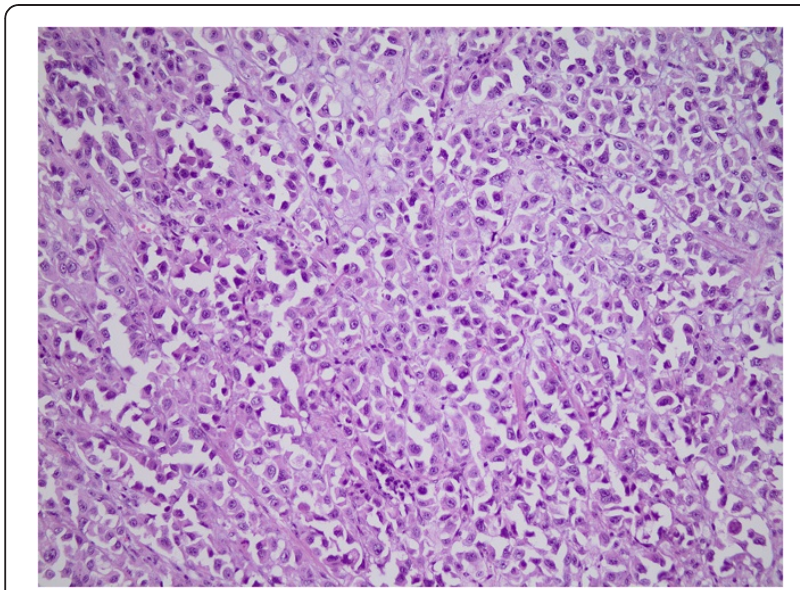

Figure 1 Hematoxylin and eosin staining (200x): Plasmacytoid urothelial carcinoma showing a characteristic single cell growth pattern with eccentrically located nuclei and abundant eosinophilic cytoplasm.

comparing the different variants of UC and their impact on survival of patients treated with cystectomy and an adjuvant cisplatin-based chemotherapy are still lacking. Thus, we describe for the first time the clinical behaviour of MPC and PUC in comparison to UC in patients treated with cystectomy and adjuvant cisplatin-based chemotherapy within the prospective and randomized trial AUO-AB05/95.

\section{Methods}

For this study 221 tissue samples of patients suffering from locally advanced bladder cancer were analysed. Of the 205 patients, 166 tissue samples from the AUO-AB05/ 95 trial comparing methotrexate, vinblastine, epirubicin, and cisplatin (M-vec) (80 patients) and cisplatin plus methotrexate $(\mathrm{Cm})$ (86 patients) were available. These patients were randomly assigned to the therapy. 39 patients with locally advanced bladder cancer were treated with radical cystectomy and adjuvant chemotherapy with gemcitabine, cisplatin (Gc) according to current guidelines were added to analysis, None of the patients received neoadjuvant chemotherapy. The study protocol of the AUO-AB 05/95 trial was approved by the committee of the "Arbeitsgemeinschaft Urologische Onkologie" of the German Cancer Society and the local ethics committees of the participating centers. All tissue samples were obtained with approval of the ethical committee of the Friedrich-Alexander-University Erlangen-Nuremberg. For this paper histological workup of tissue samples of radical cystectomy from these patients was done retrospectively from large sections by an experienced uropathologist $(\mathrm{AH})$ to identify the histologic subtypes of muscle invasive bladder cancer including determination of the histological type. Tumors were defined as variant histology (PUC and
MPC) if they showed at least $50 \%$ of the specific histology. Squamous cell carcinomas, nested-type carcinomas and small cell carcinomas were excluded from analysis. Grading was performed according to the WHO classification of 1973 and the one of 2004. The study population and details of the AUO-05/95 trial have been reported previously [9]. In addition to $178 \mathrm{UC}$ cases, 18 PUC (Figure 1) and 9 MPC (Figure 2) cases were identified. A Kaplan Meier analysis and a multivariate Cox's proportional hazards regression analysis were performed to compare overall survival between the three histologic subtypes. Statistical tests were performed using the IBM SPSS Statistics 19 software. All of the tests were two-sided, and a $P$ value $<0.05$ was regarded as statistically significant (IBM SPSS, Chicago, IL, USA). Correlations between the histological subtypes and the clinicopathological parameters were determined with the Fisher's exact test but between them and age with the $t$-test. Correlations between overall survival and histological subtypes were studied univariately with a Kaplan Meier analysis (log rank test) and multivariately with a Cox's regression hazard analysis (adjusted to age, sex, tumor grade, tumor stage, lymph node and metastases status, type of chemotherapy).

\section{Results}

Clinicopathological parameters of the included patients are shown in Table 1. Although a limited number of patients were included from the MPC and PUC tumor types, they appear to be more frequent in male patients than in female patients (UC: 3.3:1, PUC: 8:1, MPC 9:0). The mean age of the PUC patients seemed to be somewhat lower than that of patients suffering from UC or MPC (57.9, 61.0, and 62.3 years, respectively, $\mathrm{p}=0.057$ ). A

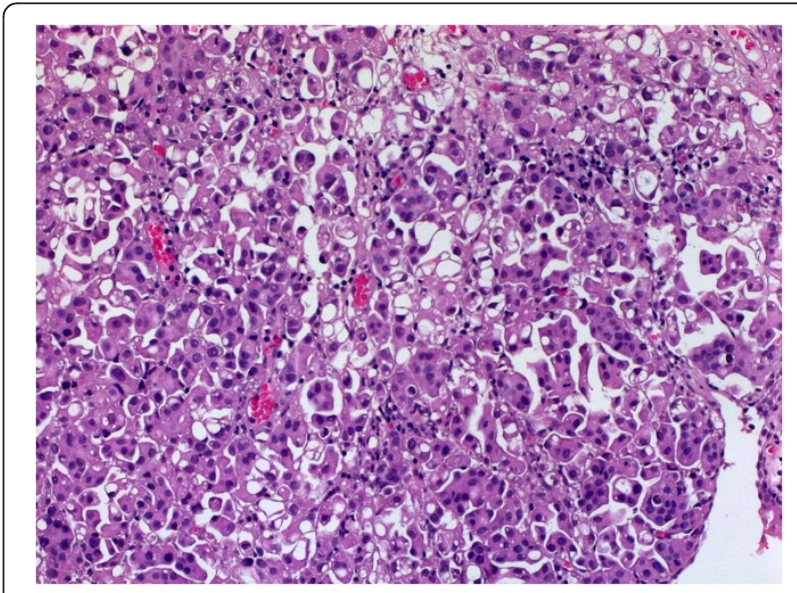

Figure 2 Hematoxylin and eosin staining (200x): Micropapillary carcinoma with medium sized tumor cells and eosinophilic cytoplasm that typically arrange in small nests and show slender, delicate processes, often with a central fibrovascular core. 
Table 1 Comparison of clinicopathological parameters of conventional UC, PUC and MPC urothelial carcinomas treated with radical cystectomy and adjuvant cisplatin based chemotherapy

\begin{tabular}{|c|c|c|c|c|c|}
\hline & Total & UC & PUC & MPC & P-Value* \\
\hline Age & & & & & $0.057^{* *}$ \\
\hline Range & $29-75$ & $29-75$ & $46-70$ & $56-70$ & \\
\hline Mean & 60.9 & 61.0 & 57.9 & 62.3 & \\
\hline Median & 61.7 & 61.9 & 59.3 & 60.8 & \\
\hline Gender & 205 & 178 & 18 & 9 & 0.116 \\
\hline Female & $45(22 \%)$ & 43 (24\%) & $2(11 \%)$ & $0(0 \%)$ & \\
\hline Male & 160 (78\%) & 135 (76\%) & 16 (89\%) & 9 (100\%) & \\
\hline pT & 205 & 178 & 18 & 9 & 0.168 \\
\hline pT1 & $5(2,5 \%)$ & $5(3 \%)$ & $0(\%)$ & $0(0 \%)$ & \\
\hline pT2 & $22(11 \%)$ & 19 (11\%) & $3(17 \%)$ & $0(0 \%)$ & \\
\hline pT3 & 142 (69\%) & $125(70 \%)$ & 13 (72\%) & $4(45 \%)$ & \\
\hline pT4 & $36(17.5 \%)$ & 29 (16\%) & $2(11 \%)$ & $5(55 \%)$ & \\
\hline Grade (1973) & 205 & 178 & 18 & 9 & 0.263 \\
\hline Grade 2 & $22(11 \%)$ & 22 (12\%) & $0(0 \%)$ & $0(0 \%)$ & \\
\hline Grade 3 & 183 (89\%) & $156(88 \%)$ & $18(100 \%)$ & 9 (100\%) & \\
\hline \multicolumn{6}{|l|}{ Grade (2004) } \\
\hline Low-grade & $0 \%$ & $0 \%$ & $0 \%$ & $0 \%$ & \\
\hline High-grade & $100 \%$ & $100 \%$ & $100 \%$ & $100 \%$ & \\
\hline $\mathrm{pN}$ & 205 & 178 & 18 & 9 & 0.743 \\
\hline pNO & $90(44 \%)$ & $80(45 \%)$ & $6(33 \%)$ & $4(44.4 \%)$ & \\
\hline $\mathrm{pN} 1$ & 45 (22\%) & $40(22.5 \%)$ & $3(17 \%)$ & $2(22.2 \%)$ & \\
\hline $\mathrm{pN} 2$ & 69 (33.5\%) & 57 (32\%) & $9(50 \%)$ & $3(33.3 \%)$ & \\
\hline pN3 & $1(0.5 \%)$ & $1(0.5 \%)$ & $0(0 \%)$ & $0(0 \%)$ & \\
\hline $\mathrm{pM}$ & 205 & 178 & 18 & 9 & 1.000 \\
\hline pMO & 204 (99.5\%) & 177 & $18(100 \%)$ & $9(100 \%)$ & \\
\hline pM1 & $1(0.5 \%)$ & 1 & $0(0 \%)$ & $0(0 \%)$ & \\
\hline Chemotherapy & 205 & 178 & 18 & 9 & 0.043 \\
\hline Gc & 39 & 39 (\%) & $0(0 \%)$ & $0(0 \%)$ & \\
\hline M-vec & 80 & $64(\%)$ & 11 (61\%) & $5(55 \%)$ & \\
\hline $\mathrm{Cm}$ & 86 & 75 (\%) & 7 (39\%) & $4(45 \%)$ & \\
\hline Survival & 205 & 178 & 18 & 9 & 0.061 \\
\hline Alive & 120 & 109 & $6(33.3 \%)$ & $5(55 \%)$ & \\
\hline Dead & 85 & 69 & $12(66.6 \%)$ & $4(45 \%)$ & \\
\hline
\end{tabular}

Abbreviations: Gc - Gemcitabine, cisplatin, M-vec - Methotrexate, vinblastine, epirubicin and cisplatin, $\mathrm{Cm}$ - Cisplatin, methotrexate.

* statistical test: Fisher's exact test.

** statistical test: $t$-test.

significant difference for chemotherapy treatment between the different histotypes $(\mathrm{P}=0.043$; Fisher's exact test; Table 1) is detected. This difference is reasoned by the fact that only UC patients but not MPC or PUC patients have been treated with gemcitabine and cisplatin. However, when we consider only $\mathrm{M}$-vec and $\mathrm{Cm}$ treatment regimens no significant difference between the different histotypes is detected ( $\mathrm{P}=0.451$; Fisher's exact test; data not shown).
Overall survival of PUC patients was significantly worse than that of patients suffering from UC or MPC (27.4 months with 95\% CI: 16.8-37.9 months, 62.6 months with 95\% CI: 54.8-70.4 months, and 64.2 months with 95\% CI: 41.9-86.4, respectively; $\mathrm{p}=0.013$ using Kaplan Meier analysis; Figure 3). A backward multivariate Cox's proportional hazards regression analysis including clinicopathological parameters 


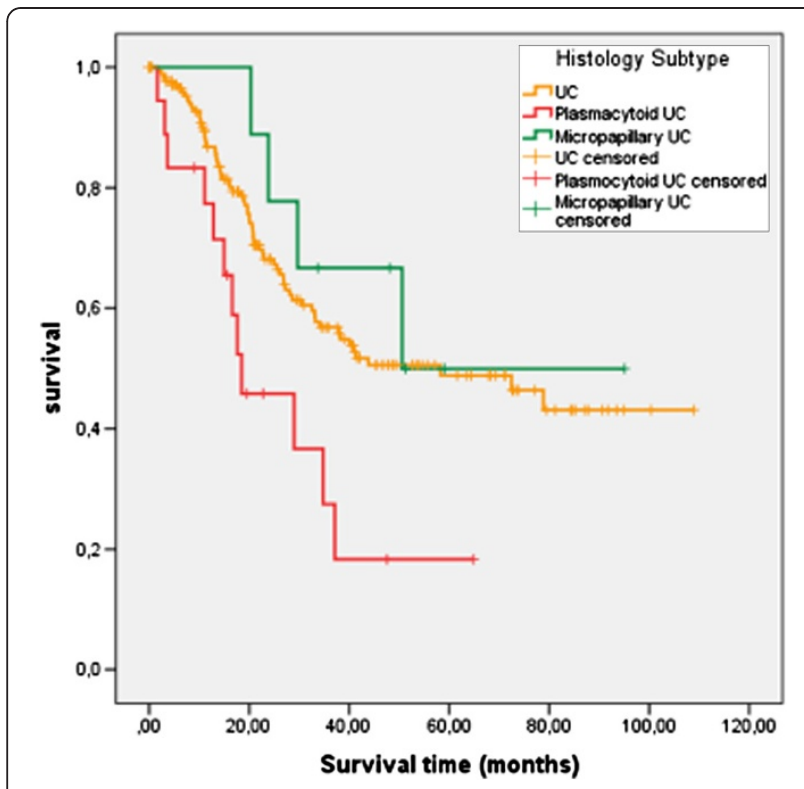

Figure 3 Kaplan-Meier analysis: Correlation of histology subtype with overall survival. Patients with a plasmacytoid urothelial cancer (lower curve, $\mathrm{N}=18$ ) showed with 27.4 months (range: 16.8-37.9) the shortest overall survival, patients with a conventional UC (middle curve, $\mathrm{N}=178$ ) survived in average 62.6 months (range: 54.8-70.4) whereas patients with a micropapillary urothelial cancer possessed the longest average survival with 64.2 months (range: 41.9-86.4; upper curve $\mathrm{N}=9$ ). The mean survival was significantly different between patients with plasmacytoid urothelial cancer and those with micropapillary urothelial cancer ( $P=0.013$; log rank test). Censoring of patients (marked with a cross) means mathematically removing a patient from the survival curve at the end of his/her follow-up time.

(age, sex, tumor grade, tumor stage, lymph node and metastases status, type of chemotherapy) showed a hazard ratio of 3.2 (95\% CI: 1.0-9.9; $\mathrm{p}=0.045)$ for PUC in contrast to patients suffering from MPC (Table 2 and Figure 4). When we analyzed the hazard ratio of the PUC patients compared with the UC patients with the same backward model we still see a 2.4-fold (95\% CI: 1.3-4.4; $\mathrm{p}=0.006$ ) increased risk of death for the PUC patients but no significant difference in risk of death between UC and MPC patients was seen (data not shown). The lymph node status was only in the first step significantly associated with the risk of death but at further backward steps only a trend towards significance was observed (Table 2).

At considering that we do not have for all patients the information for cancer-associated death we calculated their cancer-associated risk of death. In Kaplan Meier analysis we detected mean cancer-associated survival rates for PUC patients of 35.5 months, for UC patients of 67.8 months and for MPC patients of 64.2 months but this is not significant $(\mathrm{P}=0.22, \log$ rank test). In the multivariate Cox's regression hazard analysis a 2.0-fold increased risk of tumor-related death for PUC patients compared to MPC patients was found but it was not significant $(\mathrm{P}=0.26)$ and an 1.8-fold increased risk of tumor-related death for PUC patients compared to UC patients what was again not significant $(\mathrm{P}=0.15)$.

\section{Discussion}

Systemic cisplatin-based chemotherapy is regarded as the therapy of choice in metastatic UC. However, the role of adjuvant or neoadjuvant chemotherapy remains under debate $[10,11]$. Most urological and oncological guidelines recommend neoadjuvant cisplatin-based chemotherapy as the therapy of choice in locally advanced bladder cancer [12]. Because the description of several rare variants of UC of the bladder in the WHO classification of 2004, descriptions of their clinical course and the molecular features have become more prevalent. However, despite improved knowledge regarding the molecular characteristics of the histologic subtypes of bladder cancer, the impact of these differences on systemic therapies is lacking. Most patients with locally advanced UC are treated without regard to the underlying histology, although it has been reported that tumors with variant histology are associated with a higher risk of progression than conventional high grade UC [13]. As for PUC we could show in the largest series described to date, that on the one hand this subtype of UC accumulates prognostic unfavourable molecular features, like such as the loss of CK20, a high proliferation index and p53 accumulation, and as well as on the other hand exhibits characteristic molecular features, like such as a complete loss of membranous E-cadherin expression [4]. Moreover, our results from this study demonstrate that patients suffering from PUC are of younger age than those suffering from UC or MPC. However, therapeutic strategies with radical cystectomy and cisplatin-based chemotherapy does not seem to be as effective for PUC as it is described for locally advanced $\mathrm{UC}$ or MPC, even though the number of patients is limited and may serve as a bias. As it was reported recently, a complete response to adjuvant chemotherapy administering M-VEC and neoadjuvant chemotherapy using gemcitabine and cisplatin may occur at least in a subgroup of PUC -patients [14]. Therefore, chemotherapy in a neoadjuvant or adjuvant setting should be part of the therapeutic considerations. In the present study, we could not confirm these results in our analysis of adjuvant cisplatin-based chemotherapy with a median overall survival of 27.4 months, which is approximately half of that observed in the patients suffering from conventional locally advanced UC (Kaplan-Meier analysis). Thus, PUC tumor biology represents a negative prognostic factor for patients suffering from this histologic 
Table 2 Backward Cox's proportional hazards regression analysis

\begin{tabular}{|c|c|c|c|c|c|c|c|c|c|}
\hline \multicolumn{10}{|c|}{ Variables in the equation $^{b}$} \\
\hline & & \multirow[t]{2}{*}{ B } & \multirow[t]{2}{*}{ SE } & \multirow[t]{2}{*}{ Wald } & \multirow[t]{2}{*}{ df } & \multirow[t]{2}{*}{ Significance } & \multirow{2}{*}{$\begin{array}{r}\text { Exp } \\
\text { (B) }\end{array}$} & \multicolumn{2}{|c|}{ 95.0\% Confidence interval for $\operatorname{Exp(B)}$} \\
\hline & & & & & & & & lower & upper \\
\hline \multirow[t]{15}{*}{ Step 1} & age & -.151 & .232 & .422 & 1 & .516 & .860 & .546 & 1.356 \\
\hline & gender & -.046 & .281 & .027 & 1 & .870 & .955 & .550 & 1.657 \\
\hline & pT & & & .432 & 3 & .933 & & & \\
\hline & $\mathrm{pT}(1)$ & .146 & .795 & .034 & 1 & .854 & 1.157 & .244 & 5.495 \\
\hline & $\mathrm{pT}(2)$ & .047 & .464 & .010 & 1 & .919 & 1.048 & .422 & 2.602 \\
\hline & pT(3) & .185 & .307 & .364 & 1 & .546 & 1.203 & .659 & 2.196 \\
\hline & Grade & .301 & .351 & .733 & 1 & .392 & 1.351 & .679 & 2.690 \\
\hline & $\mathrm{pN}$ & -.468 & .239 & 3.842 & 1 & .050 & .626 & .392 & 1.000 \\
\hline & M & & & . & $0^{a}$ & & & & \\
\hline & Adjuvant Chemo & & & 2.790 & 2 & .248 & & & \\
\hline & Adjuvant Chemo(1) & -.359 & .538 & .446 & 1 & .504 & .698 & .243 & 2.005 \\
\hline & Adjuvant Chemo(2) & .313 & .233 & 1.806 & 1 & .179 & 1.368 & .866 & 2.160 \\
\hline & Histo subtype & & & 7.017 & 2 & .030 & & & \\
\hline & Histo subtype(1) & 1.095 & .588 & 3.463 & 1 & .063 & 2.988 & .943 & 9.466 \\
\hline & Histo subtype (2) & .261 & .535 & .237 & 1 & .626 & 1.298 & .455 & 3.703 \\
\hline \multirow[t]{10}{*}{ Step 3} & age & -.148 & .221 & .446 & 1 & .504 & .863 & .559 & 1.331 \\
\hline & gender & -.078 & .275 & .081 & 1 & .776 & .925 & .539 & 1.585 \\
\hline & Grade & .270 & .335 & .650 & 1 & .420 & 1.310 & .679 & 2.528 \\
\hline & $\mathrm{pN}$ & -.436 & .226 & 3.737 & 1 & .053 & .646 & .415 & 1.006 \\
\hline & Adjuvant Chemo & & & 2.621 & 2 & .270 & & & \\
\hline & Adjuvant Chemo(1) & -.360 & .538 & .448 & 1 & .503 & .697 & .243 & 2.002 \\
\hline & Adjuvant Chemo(2) & .294 & .229 & 1.642 & 1 & .200 & 1.342 & .856 & 2.103 \\
\hline & Histo subtype & & & 7.587 & 2 & .023 & & & \\
\hline & Histo subtype(1) & 1.150 & .581 & 3.920 & 1 & .048 & 3.157 & 1.012 & 9.852 \\
\hline & Histo subtype(2) & .298 & .523 & .325 & 1 & .569 & 1.348 & .483 & 3.759 \\
\hline \multirow[t]{9}{*}{ Step 4} & age & -.152 & .221 & .474 & 1 & .491 & .859 & .558 & 1.324 \\
\hline & Grade & .270 & .336 & .649 & 1 & .421 & 1.310 & .679 & 2.529 \\
\hline & $\mathrm{pN}$ & -.437 & .226 & 3.743 & 1 & .053 & .646 & .415 & 1.006 \\
\hline & Adjuvant Chemo & & & 2.576 & 2 & .276 & & & \\
\hline & Adjuvant Chemo(1) & -.375 & .535 & .490 & 1 & .484 & .687 & .241 & 1.963 \\
\hline & Adjuvant Chemo(2) & .285 & .227 & 1.576 & 1 & .209 & 1.330 & .852 & 2.075 \\
\hline & Histo subtype & & & 7.516 & 2 & .023 & & & \\
\hline & Histo subtype(1) & 1.155 & .580 & 3.959 & 1 & .047 & 3.173 & 1.017 & 9.895 \\
\hline & Histo subtype(2) & .317 & .519 & .372 & 1 & .542 & 1.373 & .496 & 3.797 \\
\hline \multirow[t]{8}{*}{ Step 5} & Grade & .292 & .333 & .766 & 1 & .381 & 1.339 & .697 & 2.574 \\
\hline & $\mathrm{pN}$ & -.420 & .224 & 3.522 & 1 & .061 & .657 & .423 & 1.019 \\
\hline & Adjuvant Chemo & & & 2.442 & 2 & .295 & & & \\
\hline & Adjuvant Chemo(1) & -.358 & .535 & .449 & 1 & .503 & .699 & .245 & 1.993 \\
\hline & Adjuvant Chemo(2) & .278 & .226 & 1.506 & 1 & .220 & 1.320 & .847 & 2.056 \\
\hline & Histo subtype & & & 7.388 & 2 & .025 & & & \\
\hline & Histo subtype(1) & 1.144 & .580 & 3.890 & 1 & .049 & 3.141 & 1.007 & 9.792 \\
\hline & Histo subtype (2) & .315 & .519 & .368 & 1 & .544 & 1.370 & .496 & 3.787 \\
\hline
\end{tabular}


Table 2 Backward Cox's proportional hazards regression analysis (Continued)

\begin{tabular}{|c|c|c|c|c|c|c|c|c|c|}
\hline \multirow[t]{7}{*}{ Step 6} & $\mathrm{pN}$ & -.434 & .223 & 3.771 & 1 & .052 & .648 & .418 & 1.004 \\
\hline & Adjuvant Chemo & & & 2.328 & 2 & .312 & & & \\
\hline & Adjuvant Chemo(1) & -.399 & .532 & .561 & 1 & .454 & .671 & .237 & 1.905 \\
\hline & Adjuvant Chemo(2) & .256 & .224 & 1.298 & 1 & .255 & 1.291 & .832 & 2.004 \\
\hline & Histo subtype & & & 6.980 & 2 & .030 & & & \\
\hline & Histo subtype (1) & 1.145 & .580 & 3.896 & 1 & .048 & 3.143 & 1.008 & 9.800 \\
\hline & Histo subtype(2) & .357 & .516 & .478 & 1 & .489 & 1.429 & .520 & 3.928 \\
\hline \multirow[t]{4}{*}{ Step 7} & $\mathrm{pN}$ & -.404 & .222 & 3.307 & 1 & .069 & .667 & .432 & 1.032 \\
\hline & Histo subtype & & & 8.123 & 2 & .017 & & & \\
\hline & Histo subtype (1) & 1.162 & .580 & 4.015 & 1 & .045 & 3.197 & 1.026 & 9.964 \\
\hline & Histo subtype (2) & .302 & .515 & .344 & 1 & .558 & 1.352 & .493 & 3.709 \\
\hline
\end{tabular}

a. Degree of freedom is reduced because of constant or linear dependent covariates.

b. Constant or linear dependent covariate $M=1$; Significant values are marked in bold face.

Exp(B) equals relative risk value (RR).

Abbreviations and further information.

Age: was grouped in group $1 \leq 60$ years and group $2>60$ years.

Gender: female (reference) and male groups.

pT: tumor stage; pT(1)-tumor stage 1: pT(2) tumor stage 2; pT(3): tumor stage 3; (tumor stage 4 is reference).

Grade: tumor grade: tumor grade 2 and tumor grade 3 (reference).

$\mathrm{pN}$ : lymph node status, was grouped in group1=N0 and group2 $=\mathrm{N} \geq 1$ (N1, N2, N3, reference group).

$\mathrm{M}$ : metastates status, group1without metastases (M0) and group 2 with metastases (M1), only one patient in group 2 therefore no calculations were performed.

Adjuvant Chemo: Adjuvant Chemotherapy in 3 groups: Adjuvant Chemo(1) is treated with Gc $=$ Gemcitabine, Cisplatin, Adjuvant Chemo(2) is treated with

$\mathrm{M}-\mathrm{vec}=$ Methotrexate, Vinblastine, Epirubicin, Cisplatin and the group treated with $\mathrm{CM}=$ Cisplatin, Methotrexate is the reference group.

Histo subtype: Histology subtypes in 3 groups: Histo subtype(1) is the plasmacytoid urothelial carcinoma patients' group, Histo subtype(2) is the conventional urothelial carcinoma patients' group and the group with the micropapillary urothelial carcinoma patients is the reference group.

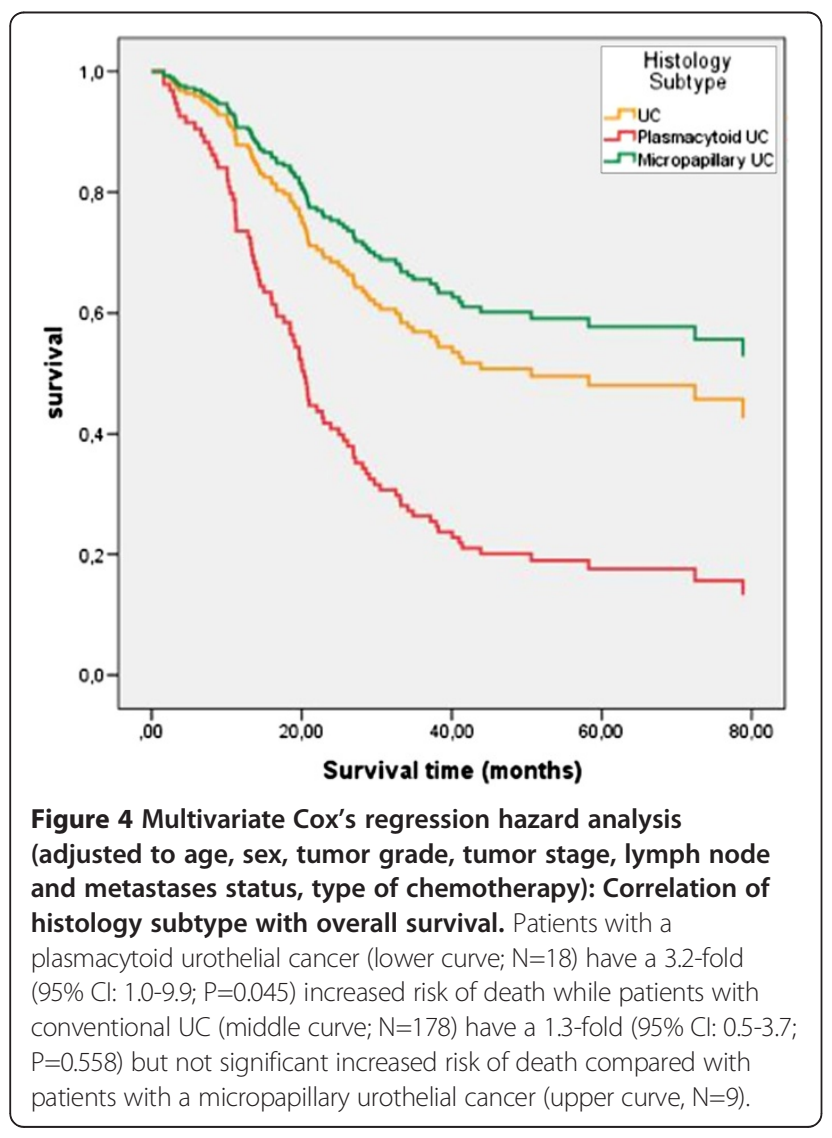

variant. Loss of E-cadherin as a sign of epithelialmesenchymal transition (EMT) and upregulation of transcriptional repressors of E-cadherin may contribute to the aggressiveness of these tumors and a possibly reduced sensitivity to chemotherapeutic agents $[4,15,16]$.

Micropapillary carcinomas have been described in different tumor entities, such as colorectal, breast, lung and others [17-19]. Thus, immunohistochemical panels for discriminating micropapillary tumors of different origins have been reported previously. Within this broad molecular panel, CK20 and uroplakin are the best molecular markers to determine urothelial origin of MPC [19]. In MPC, the initial molecular data explaining their unfavourable clinical course were recently identified, with HER2/neu gene amplification, amongst others, as a frequent molecular alteration in this variant [20]. Clinical reports suggest these are markers of biologically aggressive carcinoma with frequent lymphatic vessel invasion in TURB specimens and lymph node metastasis $[6,8,21]$. Moreover, clinical upstaging to locally advanced diseases occurs in the vast majority of the cases and represents a problem in planning therapeutic strategies [22]. In response, Compérat et al. highlighted the importance of adequate tumor sampling, including analysis of the detrusor muscle, to avoid possible upstaging. Moreover, they state that due to the associated aggressive behaviour, the proportion of micropapillary differentiation should be 
reported in all cases, even if it represents less than $10 \%$ of the specimen, as it has prognostic relevance [22]. Additionally, inter-observer reproducibility of the diagnosis of MPC is low [23], which may lead to treatment delays or the use of inappropriate therapeutic strategies adversely affecting patients' survival. Therefore, pathologists should be aware of the histologic subtypes on diagnosis. Furthermore, urologists or oncologists should take this information into account when planning surgical or chemotherapeutic treatment options. Supporting the recommendations of Compérat and coworkers Kamat et al. postulated that even papillary and non-invasive MPC should be treated by radical cystectomy to prevent progression and systemic disease [22,24]. In their analysis they demonstrated that neoadjuvant cisplatin-based chemotherapy did not result in an improved 5-year overall survival and that intravesical immunotherapy using BCG was not effective in this histologic variant [24]. Most studies on MPC describe poor disease-specific survival following adjuvant chemotherapy $[8,20,25]$. However, our data are in contrast to the experiences reported previously. We demonstrated that the survival rates were comparable for MPC and UC if treated with radical cystectomy and adjuvant chemotherapy. These contradictory results may be explained by the prospective randomized nature in which the patients were recruited and included only upon the ability to compare UC, MPC and PUC within a single trial. Although the relatively low number of patients suffering from MPC or PUC may limit the value of our study, it provides important information regarding their clinical course and the aggressive biology of the tumor subtypes. A possible limitation of our study might be the interobserver variability in defining histological subtypes as there is still no consensus on the optimal cut off value of variant histology in the specimen to define PUC or MPC. Another limitation of our results is the measurement of overall survival in our series as this could be affected by several variables besides tumor characteristics. However, on the other hand chemotherapy can have effects on comorbidity and therefore finally affect overall survival what is of relevance for the patients. Awareness of these different bladder cancer variants appears to be crucial when analyzing the molecular characteristics of advanced bladder cancers and when tailoring personalized therapeutic procedures in the future.

\section{Conclusion}

The specific tumor histology gives important prognostic information of patients suffering from locally advanced bladder cancer treated by radical cystectomy and adjuvant chemotherapy. Our results implicate that determining the exact pathological diagnosis, including the description of histologic subtypes of bladder cancers according to the WHO classification of 2004, are important. As UC, PUC and MPC are associated with a different clinical course if treated with cystectomy and adjuvant cisplatin-based chemotherapy prospective multicenter studies, comparing the different histologic variants of bladder cancer and their molecular features are necessary to tailor therapeutic strategies in the future. Furthermore, a joint study including the collection of rare bladder cancers is strongly warranted with a goal of enforcing additional molecular studies for the identification of potential prognostic and therapeutic targets.

\section{Abbreviations \\ PUC: Plasmacytoid Urothelial Carcinoma; MPC: Micropapillary Urothelial Carcinoma; UC: Urothelial Carcinoma; M-vec: Methotrexate, Vinblastine, Epirubicin, And Cisplatin; Cm: Cisplatin, Methotrexate; Gc: Gemcitabine, cisplatin; EMT: Epithelial-Mesenchymal Transition.}

\section{Competing interests}

The authors declare that they have no competing interest.

\section{Authors' contributions}

BK designed the study, interpreted the data and drafted the manuscript. BW revised the manuscript for important intellectual content and interpreted the data. $J$ and MS were involved in the data aquision and interpretation, and revised the manuscript for important intellectual content. $\mathrm{AH}$, did the histological review of the samples, reviewed the manuscript and interpreted the data. RS, FK, and SB were involved in revising the manuscript for important intellectual content as well as data interpretation. SW and HT were involved in drafting the manuscript and did the statistical analysis. All authors read, gave comments, and approved the final version of the manuscript.

\section{Note added in proof}

During the revision process Dayyani et al. published in accordance with our findings that PUC is a very aggressive variant of bladder cancer with overall poor outcomes. In addition they report that despite pathologic downstaging of patients treated with neo-adjuvant chemotherapy, relapses commonly occurred and no difference in survival between patients treated with neoadjuvant chemotherapy compared to initial surgery was observed.

\section{Acknowledgements}

We would like to acknowledge the professional editing services of American Journal Experts.

\section{Author details}

${ }^{1}$ Department of Urology, University Erlangen, Krankenhausstraße 12, Erlangen 91054, Germany. ²Department of Pathology, University Erlangen, Krankenhausstr 8-10, Erlangen 91054, Germany. ${ }^{3}$ Urology Practice, Prüner Gang, Prüner Gang 15, Kiel 24103, Germany. ${ }^{4}$ Department of Urology, Saarland University, Kirrberger Straße, Homburg/Saar 66421, Germany.

Received: 16 August 2012 Accepted: 30 January 2013

Published: 8 February 2013

\section{References}

1. Ferlay J, Parkin DM, Steliarova-Foucher E: Estimates of cancer incidence and mortality in europe in 2008. J Cancer 2010, 46(4):765-781.

2. Eble JSG, Epstein JI, Sesterhenn IA (Eds): Classification of tumours. Pathology and genetics of the tumours of the urinary system and male genital organs. Lyon: World Health Organization; 2004.

3. Keck B, Stoehr R, Wach S, Rogler A, Nolte E, Hartmann A, Wullich B: Plasmacytoid and micropapillary urothelial carcinoma: rare forms of urothelial carcinoma. Der Urologe Ausg A 2011, 50(2):217-220.

4. Keck B, Stoehr R, Wach S, Rogler A, Hofstaedter F, Lehmann J, Montironi R, Sibonye M, Fritsche HM, Lopez-Beltran A, et al: The plasmacytoid carcinoma of the bladder-rare variant of aggressive urothelial carcinoma. Int J Cancer Journal International du Cancer 2011, 129(2):346-354. 
5. Amin MB, Ro JY, el-Sharkawy T, Lee KM, Troncoso P, Silva EG, Ordonez NG, Ayala AG: Micropapillary variant of transitional cell carcinoma of the urinary bladder. Histologic pattern resembling ovarian papillary serous carcinoma. Am J Surg Pathol 1994, 18(12):1224-1232.

6. Alkibay T, Sozen S, Gurocak S, Isik Gonul I, Poyraz A, Ure I: Micropapillary pattern in urothelial carcinoma: a clinicopathological analysis. Urol Int 2009, 83(3):300-305.

7. Lopez-Beltran A, Montironi R, Blanca A, Cheng L: Invasive micropapillary urothelial carcinoma of the bladder. Hum Pathol 2010, 41(8):1159-1164.

8. Edgerton N, Sirintrapun SJ, Munoz M, Chen Z, Osunkoya AO: Micropapillary urothelial carcinoma of the urinary bladder: a clinicopathological analysis of 24 cases. Int J Urology 2011, 18(1):49-54.

9. Lehmann J, Retz M, Wiemers C, Beck J, Thuroff J, Weining C, Albers P, Frohneberg D, Becker T, Funke PJ, et al: Adjuvant cisplatin plus methotrexate versus methotrexate, vinblastine, epirubicin, and cisplatin in locally advanced bladder cancer: results of a randomized, multicenter, phase III trial (AUO-AB 05/95). J Clin Oncol 2005, 23(22):4963-4974.

10. Advanced Bladder Cancer (ABC) Meta-analysis Collaboration: Adjuvant chemotherapy in invasive bladder cancer: a systematic review and metaanalysis of individual patient data advanced bladder cancer (ABC) metaanalysis collaboration. Eur Urol 2005, 48(2):189-199. discussion 199-201.

11. Advanced Bladder Cancer (ABC) Meta-analysis Collaboration: Neoadjuvant chemotherapy in invasive bladder cancer: update of a systematic review and meta-analysis of individual patient data advanced bladder cancer (ABC) meta-analysis collaboration. Eur Urol 2005, 48(2):202-205. discussion 205-206.

12. Stenzl A, Cowan NC, De Santis M, Kuczyk MA, Merseburger AS, Ribal MJ, Sherif A, Witjes JA: Treatment of muscle-invasive and metastatic bladder cancer: update of the EAU guidelines. Eur Urol 2011, 59(6):1009-1018.

13. Shapur NK, Katz R, Pode D, Shapiro A, Yutkin V, Pizov G, Appelbaum L, Zorn $K C$, Duvdevani M, Landau EH, et al: Is radical cystectomy mandatory in every patient with variant histology of bladder cancer. Rare tumors 2011, 3(2):e22.

14. Hayashi T, Tanigawa G, Fujita K, Imamura R, Nakazawa S, Yamamoto Y, Hosomi M, Shimazu K, Fushimi H, Yamaguchi S: Two cases of plasmacytoid variant of urothelial carcinoma of urinary bladder: systemic chemotherapy might be of benefit. Int J Clin Oncol/Jpn Soc Clin Oncol 2011, 16(6):759-762.

15. McConkey DJ, Choi W, Marquis L, Martin F, Williams MB, Shah J, Svatek R, Das A, Adam L, Kamat A, et al: Role of epithelial-to-mesenchymal transition (EMT) in drug sensitivity and metastasis in bladder cancer. Cancer Metastasis Rev 2009, 28(3-4):335-344.

16. Shiota M, Song Y, Yokomizo A, Kiyoshima K, Tada Y, Uchino H, Uchiumi T, Inokuchi J, Oda Y, Kuroiwa K, et al: Foxo3a Suppression of urothelial cancer invasiveness through Twist1, Y-box-binding protein 1, and Ecadherin regulation. Clin Cancer Res 2010, 16(23):5654-5663.

17. Verdu M, Roman R, Calvo M, Rodon N, Garcia B, Gonzalez M, Vidal A, Puig X: Clinicopathological and molecular characterization of colorectal micropapillary carcinoma. Mod Pathol 2011, 24(5):729-738.

18. Siriaunkgul S, Tavassoli FA: Invasive micropapillary carcinoma of the breast. Mod Pathol 1993, 6(6):660-662

19. Lotan TL, Ye H, Melamed J, Wu XR, Shih le M, Epstein Jl: Immunohistochemical panel to identify the primary site of invasive micropapillary carcinoma. Am J Surg Pathol 2009, 33(7):1037-1041.

20. Ching CB, Amin MB, Tubbs RR, Elson P, Platt E, Dreicer R, Fergany A, Hansel DE: HER2 Gene amplification occurs frequently in the micropapillary variant of urothelial carcinoma: analysis by dual-color in situ hybridization. Modern pathology: an official journal of the United States and Canadian Academy of Pathology, Inc 2011, 24(8):1111-1119.

21. Ghoneim IA, Miocinovic R, Stephenson AJ, Garcia JA, Gong MC, Campbell SC, Hansel DE, Fergany AF: Neoadjuvant systemic therapy or early cystectomy? single-center analysis of outcomes after therapy for patients with clinically localized micropapillary urothelial carcinoma of the bladder. Urology 2011, 77(4):867-870.

22. Comperat E, Roupret M, Yaxley J, Reynolds J, Varinot J, Ouzaid I, Cussenot O, Samaratunga $\mathrm{H}$ : Micropapillary urothelial carcinoma of the urinary bladder: a clinicopathological analysis of 72 cases. Pathology 2010, 42(7):650-654.

23. Sangoi AR, Beck AH, Amin MB, Cheng L, Epstein Jl, Hansel DE, Iczkowski KA, Lopez-Beltran A, Oliva E, Paner GP, et al: Interobserver reproducibility in the diagnosis of invasive micropapillary carcinoma of the urinary tract among urologic pathologists. Am J Surg Pathol 2010, 34(9):1367-1376.
24. Kamat AM, Dinney CP, Gee JR, Grossman HB, Siefker-Radtke AO, Tamboli P, Detry MA, Robinson TL, Pisters LL: Micropapillary bladder cancer: a review of the university of texas M. D. Anderson cancer center experience with 100 consecutive patients. Cancer 2007, 110(1):62-67.

25. Heudel P, El Karak F, Ismaili N, Droz JP, Flechon A: Micropapillary bladder cancer: a review of leon berard cancer center experience. BMC Urol 2009, 9:5

doi:10.1186/1471-2407-13-71

Cite this article as: Keck et al:: Plasmacytoid variant of bladder cancer defines patients with poor prognosis if treated with cystectomy and adjuvant cisplatin-based chemotherapy. BMC Cancer 2013 13:71.

\section{Submit your next manuscript to BioMed Central and take full advantage of:}

- Convenient online submission

- Thorough peer review

- No space constraints or color figure charges

- Immediate publication on acceptance

- Inclusion in PubMed, CAS, Scopus and Google Scholar

- Research which is freely available for redistribution

Submit your manuscript at www.biomedcentral.com/submit
C Biomed Central 